\title{
A real-world assessment of mycophenolate mofetil for remission induction in eosinophilic granulomatosis with polyangiitis
}

\author{
Mariana Philobos $^{1}$ (D) Amy Perkins ${ }^{1} \cdot$ Maira Karabayas $^{1}$ (D) Paula Dospinescu ${ }^{1}$ (D) Nick Fluck ${ }^{2} \cdot$ Dana Kidder $^{2}$ (D) \\ Fiona A. Chapman ${ }^{3}$ D Neeraj Dhaun ${ }^{3}$ (D) Neil Basu ${ }^{4}$ (D)
}

Received: 5 May 2021 / Accepted: 27 July 2021 / Published online: 4 August 2021

(c) The Author(s) 2021

\begin{abstract}
Eosinophilic granulomatosis with polyangiitis (EGPA) is a form of ANCA-associated vasculitis (AAV). Clinical trials demonstrating the efficacy of mycophenolate mofetil (MMF) for remission induction in AAV excluded patients with EGPA. Despite this, MMF is commonly used in these patients. The objective of this study was to evaluate, for the first time, the effectiveness and tolerance of MMF in EGPA remission induction. A retrospective, two-center, real-world study was conducted in patients with EGPA who received MMF in addition to prednisolone for newly diagnosed or relapsing disease between 2009 and 2019. Baseline, 3-, 6- and 12-month outcome data were extracted from electronic health records. The primary outcome was disease remission, defined as a Birmingham Vasculitis Activity Score of 0 at 6 months. Secondary outcomes included disease relapse, median prednisolone dose at 12 months and drug tolerance. In total, 15 patients (73\% male, median age 57) with EGPA (11 newly diagnosed/4 relapsing) were identified. At 6 months, $67 \%$ had achieved disease remission. At 12 months, this was maintained $(66.7 \%)$ and 4 patients had relapsed. All but one patient remained on MMF at study completion and all patients tolerated MMF. Our real-world data suggest that MMF is an effective and well-tolerated agent for achieving disease remission in EGPA. A future randomized controlled trial of MMF in this neglected orphan disease is now warranted.
\end{abstract}

Keywords Mycophenolate $\cdot$ Vasculitis $\cdot$ Egpa

\section{Introduction}

ANCA-associated vasculitis (AAV) includes granulomatosis with polyangiitis (GPA), microscopic polyangiitis (MPA) and eosinophilic granulomatosis with polyangiitis (EGPA). ANCA seropositivity among EGPA patients is approximately $40 \%$, compared to up to $90 \%$ in those with GPA and MPA [1]. Moreover, the pathobiology of EGPA is distinct, dominated by eosinophils rather than neutrophilbased mechanisms [2]. Clinical trials in AAV have generally

Neil Basu

neil.basu@glasgow.ac.uk

Rheumatology Unit, NHS Grampian, Aberdeen, UK

2 Renal Unit, Aberdeen Royal Infirmary, NHS Grampian, Aberdeen, UK

3 Renal Unit, Edinburgh Royal Infirmary, NHS Lothian, Edinburgh, UK

4 Institute of Infection, Immunity and Inflammation, University of Glasgow, Glasgow, UK excluded those with EGPA. Consequently, the evidence base supporting the management of EGPA is largely inferred. The use of mycophenolate mofetil (MMF) in EGPA is one such example.

Based on expert opinion, current international clinical guidelines consider MMF as a therapeutic option for nonorgan threatening EGPA when used in combination with corticosteroids [3, 4]. MMF reduces IL-5 (the key driver of eosinophil maturation and function) generation in animal models [5] in addition to mediating inhibition of $\mathrm{T}$ and $\mathrm{B}$ lymphocyte proliferation [6]. A recent meta-analysis concluded that MMF was an effective alternative remissioninduction agent to cyclophosphamide in AAV. Although this analysis excluded patients with EGPA, it did identify those patients with myeloperoxidase (MPO) ANCA seropositivity, the prevailing ANCA in EGPA, to be specifically responsive [7]. Despite its wide spread use, no studies have evaluated the efficacy and tolerance of MMF for remission induction in patients with EGPA. Here, we describe a real-world experience of MMF use for this indication. 


\section{Methods}

A retrospective, observational study was performed. All patients diagnosed with EGPA between 2009 and 2019, and prescribed MMF (to a target dose of $1 \mathrm{~g}$ twice daily as standard) in combination with prednisolone for remission induction were identified from databases of the two largest regional vasculitis services in Scotland. These sites do not share a standardized prednisolone tapering protocol, but they generally follow a reduced dose taper, similar to that tested in the PEXIVAS trial [8], for newly diagnosed cases and a more modest starting dose (20-30 mg prednisolone) for relapsing patients. Cases were required to fulfil the 2012 Chapel Hill Consensus definition for EGPA [9].

Data were extracted from electronic health care records. Baseline characteristics (Table 1) included demographics, duration of symptoms prior to treatment, clinical manifestations, serological markers and ANCA status (Indirect Immunofluorescence (IIF) and Enzyme Linked Immunoassay (ELISA). At 0, 3, 6 and 12 months, the Birmingham

Table 1 Baseline characteristics $(n=15)$

\begin{tabular}{ll}
\hline Demography & \\
Sex M/F & $11 / 4$ \\
Age, median (IQR) & $57(27-76)$ \\
Characteristics at EGPA diagnosis & \\
Organ involvement (\%) $^{\text {Lung (including asthma) }}{ }^{\mathrm{a}}$ & $12(80 \%)$ \\
ENT & $9(60 \%)$ \\
Neuropathy & $10(67 \%)$ \\
Arthropathy & $7(47 \%)$ \\
Cutaneous & $4(27 \%)$ \\
Cardiac involvement & $2(13 \%)$ \\
Renal & $1(7 \%)$ \\
Median eosinophil $10^{9} / 1(\mathrm{IQR})$ & $3.48(0.01-21)$ \\
Positive ANCA & $7(47 \%)$ \\
BVAS, median $(\mathrm{IQR})^{\mathrm{b}}$ & $11(4-24)$ \\
\hline
\end{tabular}

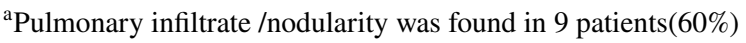

${ }^{\mathrm{b}}$ Individual BVAS is provided in supplementary table
Vasculitis activity score (BVAS, version3) [10] assessed disease activity, and prednisolone doses were calculated. In addition, 1-year relapse rates (defined as the recurrence of symptoms and signs suggestive of EGPA requiring augmentation of immunosuppression), drug in tolerance and infection rates were recorded.

The primary outcome was complete remission (defined as BVAS of 0 ) at 6 months. Secondary outcomes at 12 months were (a) complete remission, (b) partial response (a reduction of BVAS $\geq 50 \%$ compared with baseline), (c) total number of disease relapses, (d) cumulative prednisolone dose, (e) drug intolerance, (f) serum C-reactive protein, (g) peripheral eosinophil count (Table 2).

Results were reported employing summary descriptive statistics. As this project was a service evaluation exercise, the UK Health Research Authority decision tool determined that ethical approval was not needed (NHS Grampian R\&D reference 5257).

\section{Results}

Of the 15 patients (11 males) identified, 11 had newly diagnosed EGPA and 4 had relapsing disease. At baseline (Table 1), median age (IQR) was 57(27-76) and 7/15 were MPO-ANCA positive. The remaining 8 patients were ANCA negative.

Lung, nerve and ENT were the most affected organs in our cohort. Cardiac involvement, which carries poor prognosis, was evident in 2 patients.

Amongst disease relapsers $(n=4)$, previous immunosuppression included cyclophosphamide $(n=3)$, azathioprine $(n=3)$, methotrexate $(n=2)$, and rituximab $(n=1)$.

Ten patients $(66.7 \%)$ achieved the primary outcome of disease remission by 6 months, and this was maintained at 12-month follow-up. Secondary outcomes at 12 months (Table 2) revealed 4 relapses. Of these, 3 had non-organ threatening involvement (ENT or chest flares) which responded to a temporary increase in prednisolone and a longer-term increase in MMF dose (from 2 to $3 \mathrm{~g} /$ day), and one was a relapse of peripheral neuropathy, which required a switch from MMF to rituximab. All other patients remained
Table 2 Primary and secondary outcomes

\begin{tabular}{llll}
\hline & Baseline & 6 months & 12 months \\
\hline Complete remission & NA & $10 / 15(67 \%)$ & $10 / 15(67 \%)$ \\
Partial response & NA & $3 / 15(20 \%)$ & $4 / 15(27 \%)$ \\
Median cumulative prednisolone dose & NA & $3.1 \mathrm{~g}(1-5.7)$ & $4.7 \mathrm{~g}(1.9-7.9)$ \\
Total relapses & NA & $2 / 15(13 \%)$ & $4 / 15(27 \%)$ \\
Drug intolerance & NA & Nil reported & Nil reported \\
Median eosinophils count $\times 10^{9} / 1(\mathrm{IQR})$ & $3.48(0.01-21)$ & $0.2(0.02-1)$ & $0.16(0.01-0.65)$ \\
Median CRP $\mathrm{mg} / \mathrm{L}(\mathrm{IQR})$ & $13.5(<4-113)$ & $<4(1-12)$ & $<4(<4-120)$ \\
\hline
\end{tabular}


on $2 \mathrm{~g} /$ day MMF at 1 year. No patients were intolerant of MMF. Specifically, no allergic reactions were observed nor was gastrointestinal disturbance reported. Six patients experienced infections (5 respiratory, 1 urinary), one which was considered severe. All patients with baseline peripheral blood eosinophilia, recorded a normal eosinophil count at 6 and 12 months. Median prednisolone doses were 7.5 and $5 \mathrm{mg} / \mathrm{day}$ at 6 and 12 months, while the cumulative prednisolone doses were 3.1 and $4.7 \mathrm{~g}$, respectively. The prednisolone dose was increased 7 times in 7 patients.

Outcomes of newly diagnosed EGPA patients were broadly comparable to those with disease relapse, as were outcomes stratified by ANCA status where the complete remission for the positive group was achieved in $71 \%$ and $63 \%$ in the ANCA negative group (Supplementary Tables 1 and 2).

\section{Discussion}

In this retrospective evaluation, almost all newly diagnosed and relapsing EGPA patients responded to MMF in combination with prednisolone; two-thirds successfully achieving disease remission at 6 months. Moreover, after 1 year of follow-up, all patients tolerated MMF and only one patient switched immunosuppression.

This is the first study to examine the role of MMF in EGPA. In fact, very few studies have evaluated any therapy in EGPA and only mepolizumab has been tested by a randomized controlled trial. Compared to placebo, this IL-5 blocker demonstrated superior efficacy with approximately a third of patients achieving complete remission at 1 year. Although significantly less than the $67 \%$ remission rate reported in this study, the trial of mepolizumab selectively recruited relapsing or refractory EGPA patients, by definition a more treatment resistant population [11]. In keeping with this, the remission rate for those newly diagnosed $(n=11)$ in our study was even higher $82 \%$ and $73 \%$ at 6 and 12 months, respectively) and for the relapse group $(n=4)$ the remission rate was $25 \%$ and $50 \%$ at 6 and 12 months, respectively.

Another retrospective observational study, which examined the role of rituximab in EGPA [12], comprised of patients with similar baseline levels of disease activity to our study, but again included mostly relapsing/refractory patients. This, again, likely explains the apparent lower remission rates $(34 \%$ vs. $67 \%$ at 6 months and $49 \%$ vs. $67 \%$ at 12 months) and steroid sparing effects (53\% vs. $67 \%<10 \mathrm{mg}$ prednisolone at 12 months) between RTX and MMF. The median cumulative prednisolone exposure in this study ( $4.7 \mathrm{~g}$ at 12 months) is largely similar to what was observed in an open label prospective EGPA study of methotrexate and much less than older cohorts which often did not use corticosteroid-sparing agents [13].

In comparison to other AAV subtypes, our data are remarkably similar to the outcomes of the MYCYC trial. In this randomized controlled trial of MMF versus cyclophosphamide for GPA/MPA remission induction, subjects randomized to MMF achieved complete remission rates of $67 \%$ at 6 months and relapse rates of $25 \%$ at 1 year [14]. In other cohorts, relapse rates of $50 \%$ at 21 months [13] and $44 \%$ at 24 months [15] have been reported, which compares to the $27 \%$ at 12 months observed in this study.

Key study limitations should be considered. First, this is a small sample size subject to selection bias and confounding. MMF is not the only first-line remission-induction agent used by the contributing vasculitis services and the ultimate therapeutic choice was determined by individual patient factors. Generally, MMF is used for non-organ threatening presentations, although in this cohort, $n=10$ and $n=2$ manifested neuropathic and cardiac involvement, respectively. Second, this study is retrospective in design. The centralization of our electronic health records helped optimize data capture but the possibility of measurement error when computing granular outcomes, such as cumulative prednisolone dose and measuring BVAS score, cannot be excluded. Third, without a placebo comparison group, it is not possible to attribute the observed outcome improvements to MMF alone. Contextualization with historical cohorts is helpful but direct comparisons are imperfect since many of the outcomes are not identically defined. A randomized controlled trial is essential to determine true efficacy. Fourth, 1-year follow-up of subjects is adequate to evaluate remission induction but insufficient to evaluate remission maintenance.

In summary, MMF appears to be well tolerated and effective for inducing disease remission among patients with newly diagnosed or relapsing EGPA. Despite differences with GPA/MPA, these data are the first to support existing expert-based guidelines which recommend MMF as a useful remission induction option across the spectrum of AAV. Although reassuring, this is a small observational study vulnerable to confounding. Well-designed randomized controlled trials are imperative to full ascertain the role of this drug in this frequently neglected burdensome condition.

Supplementary Information The online version contains supplementary material available at https://doi.org/10.1007/s00296-021-04961-w.

Funding This study was not supported with specific funding.

\section{Declarations}

Conflict of interest The authors declare that they have no conflict of interest. 
Disclosures NB has received unrelated research funding and advisory fees from GSK and speaking fees from Roche.

Open Access This article is licensed under a Creative Commons Attribution 4.0 International License, which permits use, sharing, adaptation, distribution and reproduction in any medium or format, as long as you give appropriate credit to the original author(s) and the source, provide a link to the Creative Commons licence, and indicate if changes were made. The images or other third party material in this article are included in the article's Creative Commons licence, unless indicated otherwise in a credit line to the material. If material is not included in the article's Creative Commons licence and your intended use is not permitted by statutory regulation or exceeds the permitted use, you will need to obtain permission directly from the copyright holder. To view a copy of this licence, visit http://creativecommons.org/licenses/by/4.0/.

\section{References}

1. Durel CA, Berthiller J, Caboni S, Jayne D, Ninet J, Hot A (2016) Long-term followup of a multicenter cohort of 101 patients with eosinophilic granulomatosis with polyangiitis (Churg-Strauss). Arthritis Care Res (Hoboken) 68(3):374-387

2. Masi AT, Hunder GG, Lie JT, Michel BA, Bloch DA, Arend WP, Calabrese LH, Edworthy SM, Fauci AS, Leavitt RY et al (1990) The American College of Rheumatology 1990 criteria for the classification of Churg-Strauss syndrome (allergic granulomatosis and angiitis). Arthritis Rheum 33(8):1094-1100. https://doi.org/10. 1002/art.1780330806 (PMID: 2202307)

3. Ntatsaki E, Carruthers D, Chakravarty K, D'Cruz D, Harper L, Jayne D, Luqmani R, Mills J, Mooney J, Venning M, Watts RA, On behalf of the BSR and BHPR Standards, Guidelines and Audit Working Group (2014) BSR and BHPR guideline for the management of adults with ANCA-associated vasculitis. Rheumatology 53(12):2306-2309

4. Yates M, Watts RA, Bajema IM et al (2016) EULAR/ERA-EDTA recommendations for the management of ANCA-associated vasculitis. Ann Rheum Dis 75:1583-1594

5. Powell N, Till S, Bungre J et al (2001) The immunomodulatory drugs cyclosporin A, mycophenolate mofetil, and sirolimus (rapamycin) inhibit allergen-induced proliferation and IL-5 production by PBMCs from atopic asthmatic patients. J Allergy Clin Immunol 108:915-917

6. Bandelier C, Guerne PA, Genevay S, Finckh A, Gabay C (2009) Clinical experience with mycophenolate mofetil in systemic autoimmune conditions refractory to common immunosuppressant therapies. Swiss Med Wkly 139:41-46
7. Kuzuya K, Morita T, Kumanogoh A (2020) Efficacy of mycophenolate mofetil as a remission induction therapy in antineutrophil cytoplasmic antibody-associated vasculitis-a metaanalysis. RMDOpen 6:e001195. https://doi.org/10.1136/rmdop en-2020-001195

8. Walsh M, Merkel PA, Peh CA et al (2013) Plasma exchange and glucocorticoid dosing in the treatment of anti-neutrophil cytoplasm antibody associated vasculitis (PEXIVAS): protocol for a randomized controlled trial. Trials 14:73. https://doi.org/10.1186/ 1745-6215-14-73

9. Jennette JC (2013) Overview of the 2012 revised International Chapel Hill Consensus Conference nomenclature of vasculitides. Clin Exp Nephrol 17(5):603-606. https://doi.org/10.1007/ s10157-013-0869-6

10. Mukhtyar C, Lee R, Brown D, Carruthers D, Dasgupta B, Dubey S, Flossmann O, Hall C, Hollywood J, Jayne D, Jones R, Lanyon P, Muir A, Scott D, Young L, Luqmani RA (2009) Modification and validation of the Birmingham Vasculitis Activity Score (version 3). Ann Rheum Dis 68(12):1827-1832. https://doi.org/10. 1136/ard.2008.101279 (Epub 2008 Dec 3 PMID: 19054820)

11. Wechsler ME et al (2017) Mepolizumab or placebo for eosinophilic granulomatosis with polyangiitis. New Engl J Med 376(20):1921-1932

12. Mohammad AJ, Hot A, Arndt F et al (2016) Rituximab for the treatment of eosinophilic granulomatosis with polyangiitis (Churg-Strauss). Ann Rheum Dis 75:396-401

13. Metzler C et al (2004) Churg Strauss syndrome-successful induction of remission with methotrexate and unexpected high cardiac and pulmonary relapse ratio during maintenance treatment. Clin Exp Rheumatol 22(6 Suppl 36):S52-S61

14. Jones RB, Hiemstra TF, Ballarin J, for the European Vasculitis Study Group (EUVAS) et al (2019) Mycophenolatemofetil versus cyclophosphamide for remission induction in ANCA-associated vasculitis: a randomised, non-inferiority trial. Ann Rheum Dis 78:399-405

15. Puéchal $X$ et al (2017) Adding azathioprine to remission-induction glucocorticoids for eosinophilic granulomatosis with polyangiitis (churg-strauss), microscopic polyangiitis, or polyarteritis nodosa without poor prognosis factors. Arthritis Rheumatol 69(11):2175-2186

Publisher's Note Springer Nature remains neutral with regard to jurisdictional claims in published maps and institutional affiliations. 